\title{
Boundary layer study of a nonlinear parabolic equation with a small parameter
}

qin xulong ${ }^{1}$, xu zhao ${ }^{2}$, and wenshu zhou ${ }^{3}$

${ }^{1}$ Sun Yat-Sen University

${ }^{2}$ Beifang University of Nationalities

${ }^{3}$ Dalian Minzu University

May 8, 2021

\begin{abstract}
This paper is concerned with the initial-boundary value problem for a nonlinear parabolic equation with a small parameter. The existence of a boundary layer as the parameter goes to zero is obtained together with the estimation on the thickness of the boundary layer. The main result extends an earlier work of Frid and Shelukhin (1999).
\end{abstract}

\section{Hosted file}

QZZ-MMA.pdf available at https://authorea.com/users/412586/articles/521233-boundary-layerstudy-of-a-nonlinear-parabolic-equation-with-a-small-parameter 